\title{
FLUID FILLING-MEDIA FOR ELECTRICAL APPARATUS
}

$\mathrm{T}$ HIS paper, written by F. Meyer of the General Electric Co., was allocated for reading to the Institute of Electrical Engineers on November 23 and is now published. The author discusses the everyday fluid insulators used for electrical apparatus, particularly with transformers, and advances suggestions for improving the British Standard Specification (148, 1933, Insulating Oils for Electrical Purposes). In addition he discusses the properties of chlorinated diphenyls, perhaps the commonest noninflammable liquids used for this purpose, which are known in America under the technical name 'pyranol'. The particular requirements of oils suitable for condensers are also considered.

When oil was first adopted for filling transformers and switchgear, use was made of the best existing kind of oil. Going back no farther than thirty years ago, we find that oils were fully approved that would now be instantly rejected, both with regard to their electrical properties and their other physical characteristics. In successive editions of the British Standard Specification 148, the requirements have been tightened up until the oil which satisfies Spec. 148 could be safely used medicinally. Unfortunately the demand of the transformer manufacturers was for an oil based on its sludge value and tested by a method far removed from service conditions, and very little attention was paid to its other properties. It is a well-known fact that the oxidation products of an oil at $150^{\circ} \mathrm{C}$. are very different from those taken at $90^{\circ} \mathrm{C}$. both in quantity and character. As a test for the eradication of unsuitable oils the author thinks that the Michie test has outgrown its original use.

Fifty per cent of the transformer oil used to-day is Grade $A$ oil, typical examples of which give practically zero sludge values. For switchgear purposes where sludge is of small importance, highly refined oil is quite unnecessary. The less refined also the oil the cheaper it is, a point of importance when we consider the large bulk of oil used. Class $B$ oils, which formerly were just obtainable with sludge values between 0.7 and 0.8 , are to-day readily procurable at the low figure of $0 \cdot 3$ or even less. The author points out that in bringing the sludge value of oils down to these low limits the danger of the development of acidity is now as menacing as the old danger of high sludge values was. It may well be that in the light of modern experience, the highly refined $\boldsymbol{A}$ oils employed so widely to-day which have practically zero sludge value will be discarded and an oil of a moderate degree of refinement, but low developed acidity, will become the standard of transformer oil. Tests of $A$ and $B$ oils taken over a period of six months show that the rate of acidity increases for both oils, for the first three months is much the same but for the next three months the average acidity of the $A$ oil increases much more rapidly than the $B$ oil.

The greatest drawback of oil as a filling medium is its inflammability. In consequence of recent disastrous fires at Bradford and Kingston due to the oil in transformers and switchgear, and also the danger of fire from incendiary bombs, engineers have been recently studying closely the only noninflammable liquids at present available, that is, synthetic chlorinated compounds. The commonest of these are the chlorinated diphenyls made in America and on the Continent but not yet manufactured in Great Britain. The degree of inflammability of pure chlorinated diphenyl, generally called in Great Britain 'aroclor', depends on the degree of chlorination. Experiments demonstrate not only the non-inflammability of 48 per cent chlorinated aroclor but also that, in addition, it has the valuable property of acting as a fire extinguisher.

In conclusion, the author discusses condenser oils, which are generally pure mineral hydrocarbon oils. Service conditions in this case are so severe that they cannot be used as delivered by the oil refiners, which can safely be done with transformers.

\section{ROAD SURFACE TEXTURE PRINTS}

\begin{abstract}
$\mathrm{T}$ HAT surface texture is an important factor in determining the resistance of a road to skidding has long been recognized, and at the Road Research Station one of the studies which has received attention has been the development of a satisfactory method of recording this quality. The latest device is an adaptation of the method of offset printing, and it is also the simplest and the most revealing in that it readily lends itself to a study of the changes which oecur during the life of the road carpet and of its progress towards failure. A few square inches of the road under examination are cleaned of dust and then carefully inked over either by an ordinary half-tone black printing ink diluted with paraffin or by a water letterpress ink. A clean rubber roller, of specified hardness, is then run over the inked area and the impression taken by the roller is transferred to a sheet of white paper.
\end{abstract}

The prints which are obtained in this way are remarkable for the wealth of detail which they exhibit, even the finest particles on the surface, about a thousandth of an inch in diameter, being recorded. A Bulletin-Road Research Bulletin No. 3 ("A Printing Method of Recording Road Surface Texture". H.M. Stationery Office. 9d.)-just issued by the Department of Scientific and Industrial Research and the Ministry of Transport; gives a description of the process and of the apparatus which, it may be mentioned, packs into a suit-case. It exhibits a number of reproductions of prints taken by the process and these show the stones in the road surface appearing as in relief, the shading of the ink giving a stereoscopic effect. The interpretation of the prints requires experience, and though a surface likely to be slippery is easily recognizable, the assessment of the relative slipperiness of various carpets from their texture prints is more difficult. A series of these prints exhibits the changing nature of a given road surface; the prints are very convenient both for comparison and for storage. 